\title{
DESIGN OF AN ULTRA-WIDEBAND GROUND-PENETRATING RADAR SYSTEM USING IMPULSE RADIATING ANTENNAS
}

\author{
J.B. Rhebergen and A.P.M. Zwamborn \\ D.V. Giri \\ TNO Physics and Electronics Laboratory, \\ The Netherlands. \\ Pro-Tech, \\ United States of America.
}

\begin{abstract}
At TNO-FEL, one of the research programs is to explore the use of ultra-wideband (UWB) electromagnetic fields in a bi-static ground-penetrating radar (GPR) system for the detection, location and identification of buried items of unexploded ordnance (e.g. land mines). In the present paper we describe the current status of the development of this system.

The UWB ground-penetrating radar system is designed to operate in the frequency band from $200 \mathrm{MHz}$ to $3 \mathrm{GHz}$ and uses impulse radiating antennas (IRAs) as transponders to radiate and receive very short electromagnetic pulses from a short distance above the soil. The receiving IRA is similar to the transmitting IRA and is connected to a receiving unit which comprises of an attenuator, time gate switch, trigger delay generator and a sampling oscilloscope (see figure 1). A personal computer is used to control the equipment. In order to perform controlled radar experiments, a full-size experimenting and testing facility has been erected on the premises of TNO-FEL.
\end{abstract}

\section{SYSTEM DESIGN}

To design an UWB GPR system for detection, location and identification of land-mines the following points have to be successfully combined.

- generation of UWB EM field (i.e. source)

- radiation of UWB EM field (i.e. antenna)

- characterization of the soil and air/earth interface

- reception and synthesis of scattered fields

- signal processing techniques and identification of objects

- controlled experiment/test environment.

This paper concentrates mainly on the system design. The signal processing aspects are, for the time being, saved for a future follow-up paper although some preliminary work has taken place [1]. An overview of the overall system concept is shown in figure 1. The target volume is illuminated by an ultra-wideband electromagnetic field, with an angle of incidence chosen to maximize energy transmission into the ground, whilst minimizing reflection from the ground/air interface. Presently, the main interest

is in objects varying in size from about $5 \mathrm{~cm}$ up to ca. $75 \mathrm{~cm}$. In air this corresponds to wavelengths of $1.5 \mathrm{~m}$ to $0.1 \mathrm{~m}$. Consequently the frequency range of interest extends from $200 \mathrm{MHz}$ up to $3 \mathrm{GHz}$.

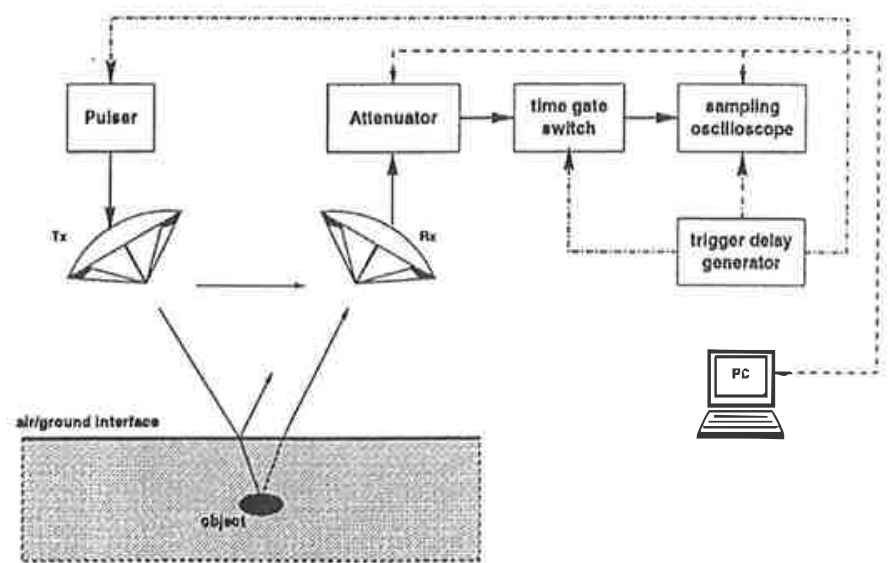

Figure 1- Schematic block diagram of a generic UWB GPR system

The principal goal is to perform the needed analytical and design work to support the construction of an experimental/test facility, where targets can be buried in a sand medium and 
illuminated by an ultra-wideband electromagnetic field. The scattered fields have to be observed and diagnosed for the purpose of building an electronic database containing the responses of a large number of buried objects.

\section{Generation of UWB EM fields}

For our UWB GPR system it was decided to use a solid state pulse generator with a double exponential voltage waveform. Such a waveform can be modeled by,

$V(t)=V o\{\exp (-\beta t)-\exp (-\alpha t)\} u(\mathrm{t})$,

with $V_{0,}, \alpha$ and $\beta$ known constants, $\alpha>\beta$ and $u(t)$ the Heaviside step function. In our case the parameters are

$$
\begin{aligned}
& V_{o}=9.635 \mathrm{kV}, \\
& \alpha=2.10^{10} \mathrm{~s}^{-1} \text { and } \beta=2.5 \cdot 10^{8} \mathrm{~s}^{-1}
\end{aligned}
$$

This gives rise to a Fourier spectrum,

$$
\bar{V}(j \varpi)=V_{0}\left[\frac{1}{j \varpi+\beta}-\frac{1}{j \varpi+\alpha}\right] \text {. }
$$

Since the magnitude of the far field is proportional to the derivative of the voltage waveform, a better radiated spectrum (enhanced bandwidth) is obtained when compared to sinusoidal mono-cycles. The pulser is designed to drive a $50 \Omega$ load, is fully protected agianst open and shortcircuit loads and has a pulse repetition frequency of $1 \mathrm{kHz}$. The pulse has a $10 \%$ to $90 \%$ rise time of about 100 ps and a fullwidth half-maximum (FWHM) time of 3 ns.

\section{Radiation of UWB EM fields}

Many commercially available wideband antennas have poor performance when it comes to radiating very short pulses. Part of the UWB GPR system design entailed the design of an IRA for ground penetrating radar applications. The upper frequency limit $f_{\text {upper }}$ of such an antenna is determined by the pulse rise time $t_{\text {rise }}$ while the lower frequency limit $f_{\text {lower }}$ is determined by the dimensions of the reflecting dish. Pulse rise times of 25 ps and upper frequency limits of $20 \mathrm{GHz}$ have been demonstrated in IRA designs. Experiments have shown that an antenna with a $4 \mathrm{~m}$ reflecting dish and a $60 \mathrm{kV}$ switch with a 85 ps rise time has a bandwidth extending from $35 \mathrm{MHz}$ to $4 \mathrm{GHz}$ with a field stength of $4 \mathrm{kV} / \mathrm{m}$ at $300 \mathrm{~m}$. The frequency range of interest in our case is from about $200 \mathrm{MHz}$ to 3
$\mathrm{GHz}$. Hence the diameter of the reflecting dish was proposed to be in the order of $1 \mathrm{~m}$.

\section{Soil chracterization}

Electromagnetic waves are damped considerably when traveling through a medium with a conductivity greater than zero. In our experimental set-up the angle of incidence and incident polarization are chosen such as to maximize the energy transfer from the transmitter into the ground. This is based on the technique of pulse matching into the ground developed in [10], for vertical polarization. If frequencies are much larger than the relaxation frequency of the ground $\left(f_{\text {rela. }}=\sigma / \varepsilon\right)$, there is an angle of incidence at which there is very little reflection of the incident wave from the air/ground interface. This is called the highfrequency Brewster angle. For $f \gg f_{\text {relax }}$ it can be shown that reflection is minimal (theoretically zero) when,

$$
\tan \left(\psi_{\mathrm{B}}\right)=\sqrt{ } \varepsilon_{\mathrm{r}}
$$

When $\sigma=0.01 \mathrm{~S} / \mathrm{m}$ and $\varepsilon_{\mathrm{r}}=10$, then the relaxation frequency of the ground $f_{\text {relax }}=113 \mathrm{MHz}$. Hence when the conductivity $\sigma \leq 0.01 \mathrm{~S} / \mathrm{m}$, which is quite typical, the radiated spectrum of the IRA will be transmitted into the ground with minimum reflection. The next table shows some examples of high-frequency Brewster angles at various values of permittivity.

\begin{tabular}{|l|ccccc|}
\hline$\varepsilon_{\mathrm{r}}$ & 2 & 4 & 6 & 8 & 10 \\
\hline$\psi_{\mathrm{B}}$ & $54.73^{\circ}$ & $63.42^{\circ}$ & $67.79^{\circ}$ & $70.53^{\circ}$ & $72.45^{\circ}$ \\
\hline
\end{tabular}

\section{Reception and synthesis of scattered fields}

The receiving system consist of:

- receiving antenna, similar to transmitting antenna;

- processing unit (data acquisition)

The processing unit has to perform two tasks:

- recording and preprocessing the incoming signal, reducing noise, clutter and distortion caused by the signal path;

- perform some signal processing on the scattered field in order to be able to identify the buried objects.

Data acquisition and processing is done by a sampling (digitizing) oscilloscope able to perform measurements on signals with bandwidth of up to 20 GHz. The input signal cannot be measured reliably 
at just one instance, which is why a sampling oscilloscope employs a repetitive sampling architecture. This in turn requires the transmitted pulse to be repetitive as well. Arithmetic averaging of the repetitive input signal can significantly reduce the noise (and jitter) on the received scattered field signal. Gating allows isolation of the part of the signal due to the buried objects.

\section{Signal processing and identification}

Until 1980, almost all of the work in the field of groundpenetrating radar was directed towards the detection of buried objects. Only very few attempted the problem of target identification (or classification), which is a problem far more severe than the identification of aerospace targets by conventional radars where the target can literally be seen and the class of false targets is limited in scope. Underground there are varieties of undesired targets that complicate the task. Furthermore, the ground medium involved, is usually lossy, inhomogeneous and electrically weather-dependent. These problems, together with the presence of the air-ground interface, makes the task of subsurface target identification truly formidable.

It was suggested by some authors that the socalled singularity expansion method (SEM) is one of the few methods capable of performing identification of objects [2]. This method is based on the theoretical observation that all objects have natural resonant frequencies that depend on their size, shape and the material decomposition only and not on the orientation of the object or on the direction of the incoming field. When a target is hit by a short electromagnetic pulse, the target will "ring" and radiate a scattered field that contains as prominent constituents a set of damped sinusoids. These sinusoids represent the natural resonant frequencies. The bandwidth of the ultra-wideband system must be large enough to cover all the resonant frequencies of interest. The SEM will be exploited in the current problem of classification of the buried object for the calculation of the internal resonances of the buried object by processing the reflections or backscattering from the objects. Some initial analysis with synthetic data has already been performed [1].

\section{DESIGN AND CONSTRUCTION OF AN IRA}

The paraboloidal reflector antenna fed by a pyramidal horn has found wide-spread application in radar and communication engineering. The reflector antenna also has very useful characteristics when it is fed by two or four conductor transmission lines. A dispersion-less wideband antenna with a nearly flat radiating spectrum is desirable for short pulse applications. The reflector IRA employs a paraboloidal reflector fed by TEM lines [11, 12], and is an example of an aperture antenna. It is well known that the radiated field from an aperture antenna consists of a spatial integration of the aperture fields over the aperture, while the temporal behaviour of the aperture field is differentiated in the far field. In a practical situation, the illuminating field or the aperture field is a double exponential waveform and the radiated field then becomes impulse-like with a very large bandwidth ratio.

The reflector IRA under consideration consists of

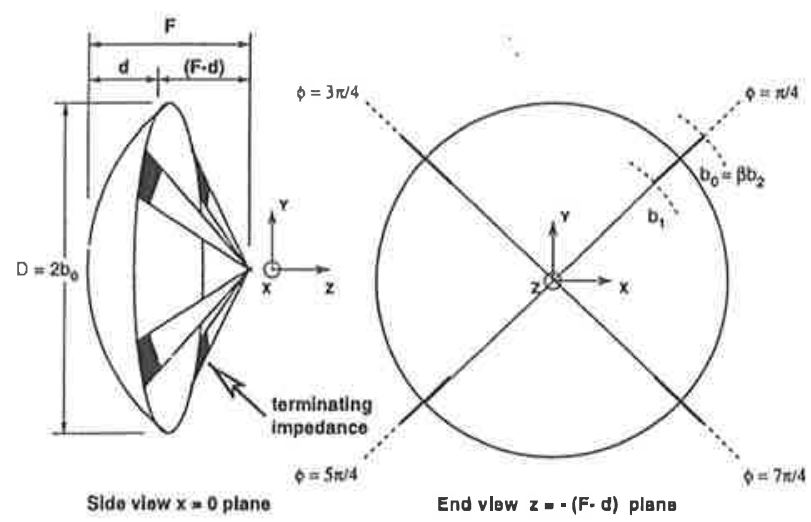

Figure 2 - An illustration of a reflector fed by a pair of coplanar conical TEM lines.

a paraboloidal reflector fed by two pairs of coplanar feed plates as illustrated in figure 2. Coplanar feed plates are chosen over the more conventional facingplate geometry to minimize the aperture blockage effects. To reduce the aperture blockage, the feed plates are required to be narrow, resulting in feed impedances of several hundreds of Ohms. The 400 $\Omega$ lines are connected in parallel resulting in a net feed impedance of $200 \Omega$. The aperture area should be as large as practically possible, since the far field is proportional to the square root of this area for a constant voltage at the feed. The magnitude of the far field is proportional to the aperture area for a constant aperture field. The pulse generator has to be of the differential type to avoid common mode currents on the feed plates, which could distort the desired features in the far field. The driving voltage is $V(t)=\left(V_{0} / 2\right) u(t)$, where $V_{0}$ is already.defined in eqn. 2. Since $E_{f a r} \propto \partial V / \partial t$, it; is desirable to maximize this rate of rise of the incident field or the voltage pulse. 
Two identical reflectors were manufactured with the following characteristics.

- one piece, spun aluminum, paraboloidal surface

- diameter $D=900 \mathrm{~mm}$

- focal length $F=337.5 \mathrm{~mm}$

- profile accuracy $\leq 1.5 \mathrm{~mm}$

- $f_{d l}=F / D=0.375$

The desired rise time of 190 ps implies an upper 3 $\mathrm{dB}$ frequency of $3.5 \mathrm{GHz}$ which gives rise to wavelengths in air of $85.7 \mathrm{~mm}$. The surface tolerance of the reflector is small compared to the shortest wavelength and hence it is acceptable.

Next, we look at an estimation of boresight waveforms. For analysis purposes, one could consider a single (two conductor) coplanar feed, although in practice we used two such feed lines connected in parallel for a more uniform illumination of the reflector (see figure 2). When the reflector IRA was originally proposed [5], the boresight radiation was predicted to consist of a feed step followed by an impulse-like behaviour. It was also shown that the total area under these two parts of the radiated waveform (i.e. prepulse plus impulse) is zero. This means, that there is no DC component in the radiated waveform consisting of the prepulse and the impulse. This further implies that the portion of the radiated waveform after the impulse must have a net zero area in itself. The post impulse portion consists of diffracted signals from the feed plate and the circular rim of the paraboloidal reflector. A more recent analysis [6] has extended this result by chronologically considering the various temporal elements of the boresight radiation, which is illustrated in figure 3 . Let us assume that the voltage pulse generator is switched on at $t=0$, and the observer is at a distance $r(=z)$ to the right of the focal point of the paraboloid. These temporal elements are:

- Prepulse: feedstep $E_{y^{\prime} 1}(r, t)$

- Main pulse: impulse $E_{y^{2}}(r, t)$

- Postpulse:

- feedplate diffraction: $E_{y 3}(r, \mathrm{t})$ (actually this consists of two parts originating form the plate edge (3a) and the plate itself (3b))

- edge diffraction from circular rim of the reflector, $E_{r \cdot 4}(r, t)$

- Entire pulse constraints: low-frequency dipole moment radiation and no radiation at zero frequency (DC).

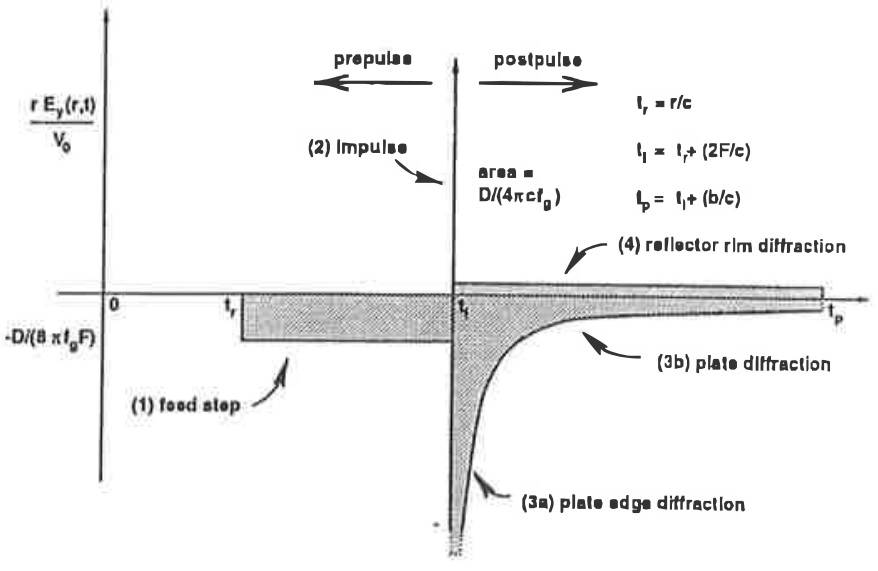

Figure 3 On-axis radiation from a canonical reflector IRA.

The far field $\vec{E},(\vec{r}, t)$ is given by,

$$
\begin{aligned}
\vec{E}_{f}(\vec{r}, t) & =\vec{l}_{y} E_{y}(r, t), \quad \text { where } \\
E_{y}(r, t) & =\sum_{\mathrm{i}=1}^{4} E_{y \mathrm{i}}(r, t)
\end{aligned}
$$

+low-frequency radiation from dipole moments resulting in time-integral constrains on entire pulse.

Detailed, closed-form expressions for the components $E_{y i}(r, t)$ in the above expression, are available in [7]. The results of eqn. 6 is illustrated in figure 3 . The time-integral constraints imposed by the low-frequency radiation are,

i) the complete first-time integral of the radiated waveform must be zero, and

ii) the second-time integral must be proportional to the late-time dipole moments.

\section{Feed plates, terminating and matching}

The feedplates form a parallel combination of $2 \mathrm{x}$ $400 \Omega$ conical TEM lines, giving the IRA an input impedance of $200 \Omega$. Matching to the $50 \Omega$ pulse source is done by means of balun. Suitable highfrequency, high-voltage, and high-impedance cables are difficult to find. Finally a $100 \Omega$ flexible cable with a PTFE dielectric was chosen. The latter is bound to introduce some dispersion, however the cable can be replaced by a better one in the future. Terminiation of each feed plate at the dish edge consists of a net DC impedance of $200 \Omega$. The insulating spacer is a high density polyethylene slab 
the dimensions of which are optimised experimentally. Capacitance of the slab is trimmed by drilling holes (tuned with help of TDR measurements at the antenna terminal). The terminating resistor network, consist of a seriesparallel network ( 5 by 5 ) of $1 \mathrm{~W}, 200 \Omega$ carbon composite resistors of $10 \%$ tolerance. Thus total heat dissipation is not a problem. Since the length of the resistor chain is $\geq 10 \mathrm{~cm}$, the voltage stand-off (in the order of $10 \mathrm{kV} / \mathrm{cm}$ ) is not seen to be a problem either.

\section{Far field of the IRA}

If an aperture antenna of diameter $D$ is illuminated by a CW field of frequency $f$, then the far field is determined by the distance $r$ such that,

$$
r \geq \frac{2 D^{2}}{\lambda}
$$

which is obtained by requiring that $\Delta_{r} \leq(\lambda / 16)$ where $\Delta_{r}$ is the path difference of the edge ray and the central ray from aperture to observer. For pulsed antennas one can define a clear time $t_{c}=\Delta_{r} / c$ and require that $t_{c}<t_{\text {rise time }}$ of the incident pulse. It is seen that

$$
t_{c}=\frac{1}{c}\left[\sqrt{\frac{D^{2}}{4}+r^{2}-r}\right] .
$$

Requiring that $t_{c}<t_{\text {rise time }}$, it is observed from table 1 that the observation point has to be greater than $8 \mathrm{~m}$, to be in the far field, by a comparison of the clear time with the rise time of $100 \mathrm{ps}$. The radiated fields on the bore-sight are known in closed form in the far field [5, 6, 7, 8, 9]. More recently, Russian researchers [14] have worked out near and far field expressions of the E-field on the bore-sight of an IRA. Using these expressions, we have estimated the on-axis E-field at various distances in the near and far field of the antenna. This is the subject of the next subsection. Although $8 \mathrm{~m}$ is the minimum distance to be in the far field, the actual experiments of illuminating targets can be performed at shorter distances in the near field.

The pulse is broader and has more low-frequency components in the near field. In fact, very near the Table 1. Clear time $t_{c}$ for a reflector diameter $\mathrm{D}=0.9 \mathrm{~m}$

\begin{tabular}{cccc}
\hline $\begin{array}{c}r \\
\text { (in } \mathrm{m} \text { ) }\end{array}$ & $\begin{array}{c}r+\Delta_{r} \\
\text { (in } \mathrm{m} \text { ) }\end{array}$ & $\begin{array}{c}\Delta_{r} \\
\text { (in } \mathrm{mm} \text { ) }\end{array}$ & $\begin{array}{c}t_{c}=\Delta_{r} / \mathrm{c} \\
\text { (in } \mathrm{in} \text { ) }\end{array}$ \\
1.0 & 1.096 & 96 & 320.0 \\
2.0 & 2.050 & 50 & 166.6 \\
2.5 & 2.540 & 40 & 133.3 \\
3.0 & 3.033 & 33 & 110.0 \\
3.5 & 3.529 & 29 & 96.6 \\
4.0 & 4.025 & 25 & 83.3 \\
5.0 & 5.020 & 20 & 66.6 \\
8.0 & 8.012 & 12 & 40.0 \\
10.0 & 10.010 & 10 & 33.3 \\
& & &
\end{tabular}

the antenna, for example at the feed point, the electric field is similar to the voltage pulse which is a double exponential waveform. The differentiation occurs in the far field and the transformation from near to far field is gradual and not abrupt. This transformation can be experimentally observed by measuring the electromagnetic field on-axis, as one moves away from the focal region. These values

Table 2. Near field and far field characteristics of the IRA (bore-sight)

\begin{tabular}{lll}
\hline quantity & near field & far field \\
$======================$ \\
distance $R$ & $5 \mathrm{~m}$ & $10 \mathrm{~m}$ \\
cleartime & $66 \mathrm{ps}$ & $33.3 \mathrm{ps}$ \\
prepulse & $-255 \mathrm{~V} / \mathrm{m}$ & $-127 \mathrm{~V} / \mathrm{m}$ \\
prepulse duration & $2.25 \mathrm{~ns}$ & $2.25 \mathrm{~ns}$ \\
impulse peak & $5.9 \mathrm{kV}$ & $3.8 \mathrm{kV}$ \\
impulse duration & $85 \mathrm{ps}$ & $60 \mathrm{ps}$ \\
& & \\
\hline
\end{tabular}

have been experimentally verified during initial measurements and compare favourably with the above table. Expressions for the radiated spectrum are available in literature as mentioned earlier. Calculations for our IRA results in the values tabulated in table 3.

Table 3. Calculated bore-sight values for a distance $R=10 \mathrm{~m}$

$$
\begin{array}{ll}
E & =2.85 \cdot 10^{7} \mathrm{~V} / \mathrm{m}(+/-12 \%) \\
f_{l}(3 \mathrm{~dB}) & =80 \mathrm{MHz} \\
f u & =10 \mathrm{GHz} \\
\text { BW-ratio } & =125 \\
\text { decades } & =2.097
\end{array}
$$

Measurements indicate that due to practical restrictions the actual performance is not quite as good as theoretically expected. 


\section{UWB GPR EXPERIMENTAL FACILITY}

To be able to test the newiy developed UWB GPR system and to perform experiments in il controlled environment. a full scale experimental/test facility wals erected on the premises of TNO-FEL. This facility has been developed in cooperation with the Delft University of Technology and will be used to illuminate objects buried in a realistic sand medium with an ultra-tvideband electromagnetic field. The electromagnetic field scattered by the buried object has to be recorded and diagnosed for the benefit of developing a computer database containing the responses of a large number of potential targets (i.e. landmines etc.).

The experimental facility consists of a buried wooden box. The dir.. nsions of this box are $10 \mathrm{mx}$ $10 \mathrm{~m}$ wide and $3 \mathrm{~m}$ deep. Special care has been taken not to use any metal parts in the construction of the box or in the vicinity thereof. The sand-box is filled with clean homogeneous river sand. In order to keep the condition of the sand in the box optimal and to prevent pollution from the outside (for example ground water) entering the box, a drainage system was installed and the inside of the box was covered with a watertight plastic lining. To prevent the weather from influencing the test conditions and to protect the measuring equipment, a large tent covers the entire site. While filling the box with sand, special care was taken to get a homogeneous profile. Current tests indicate that this was not entirely successtul but should be good enough to work with. Later on we might choose to empty the box and refill it with a ground (soil) of a different composition. To facilitate the measurement of EM transmissions into the ground a square PVC tube running from the surface of one side to the bottom of the other side has been installed about one meter from the edge of the sand-box.

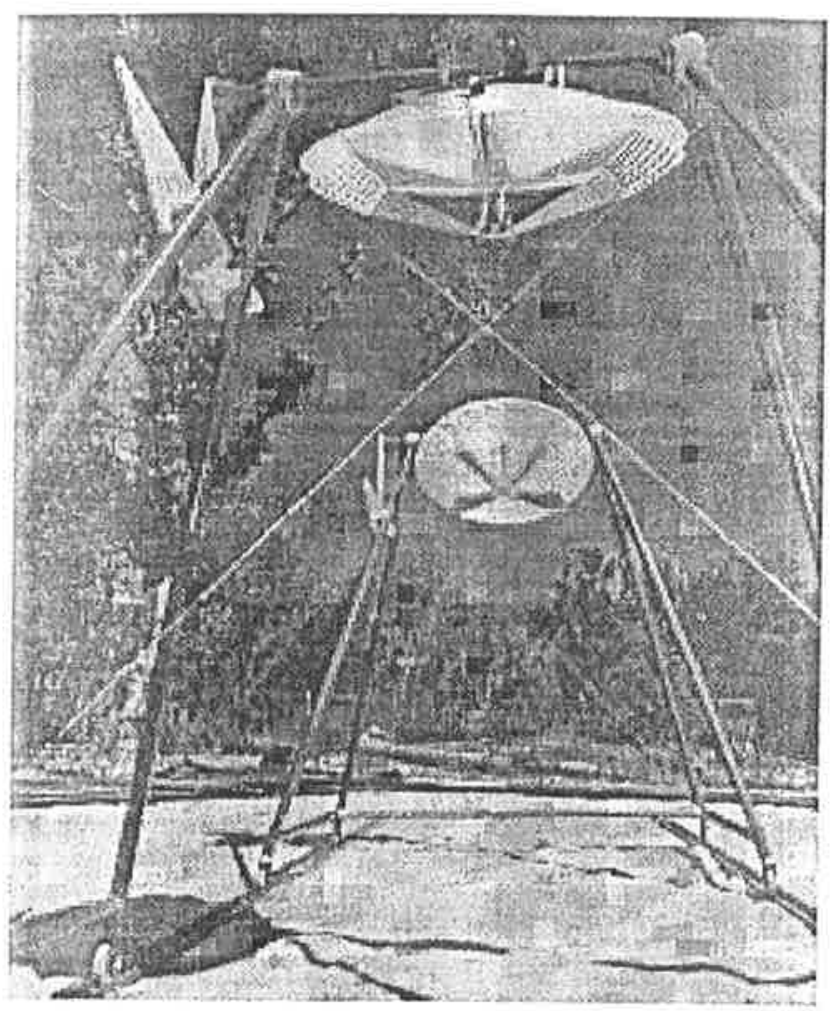

Figure 4. The finished IRAs mounted on A-frames at the UWB GPR experimental facility:

\section{REFERENCES}

[1] J. Lo Vetri, S. Primak, B.J.A.M. van Leersum and A.P.M. Zwamborn, June 1998, "Feasibility study into the identification of land-mines using UWB radar and analysis using synthesized data", presented at EUROEM'98, Tel Aviv, to be published in "Ultra-Wideband Short-Pulse Electromagnetics 4"

[2] C.E. Baum, E.J. Rothwell, K.M. Chen and D.P. Nyquist, 1991, "The Singularity Expansion Method and its application to target identification", Proc. of the IEEE, vol.79, no.10, pp. 1481-1492

[3]C.E. Baum,1994, "Signature-based target identification and pattern recognition", IEEE Antennas and Propagation Magazine, vol.36, no.3, pp. 44-51

[4]C.E. Baum,L. Carin and A.R Stone, 1996, "Ultrawideband short-pulse electro-magnetics 2", Plenum Press, New York, ISBN 0-306-45502-X.

[5] D.V. Giri and C.E. Baum, "Temporal and spectral radiation on bore-sight if a reflector type of impulse radiating antenna (IRA)", published in [4].

[6]D.V. Giri and C.E. Baum, November 1989, "Radiation of impulse-like transient fields", 
Sensor and Simulation Notes, no.321.

[7] D.V. Giri and C.E. Baum, February 1994. "Reflector IRA design and bore-sight temporal waveforms", Sensor and Simulation Notes, no. $365^{\prime}$

[8] D.V. Giri, H. Lackner, I.D. Smith, D.W. Morton, C.E. Baum, J.R. Marek, D. Scholfield and W.D. Prather, July 1995, "A reflector antenna for radiating impulse-like waveforms", Sensor and Simulation Notes, no. 382

[9] D.V. Giri, November 1995, "Radiated spectra of impulse radiating antennas (IRAs)", Sensor and Simulation Notes, no. 386

[10] C.E. Baum, March 1967, "The Brewster Angle Wave Matcher", Sensor and Simulation Notes, no. 37

[11] C.E. Baum, D.V. Giri and R.D. Gonzalez, April 1976, " Electromagnetic field distribution of the TEM mode in a symmetrical two-parallel plate transmission line", $\underline{\text { Sensor and Simulation Notes }}$ no. 219

[12] F.C. Yang and L. Marin, September 1977, "Field distribution on a two-conical plate and a curved cylindrical plate line", Sensor and Simulation Notes, no.229

[13] J.J.A. Klaasen, August 1992, "An efficient method for the performance analysis of boundedwave nuclear EMP simulators", Sensor and Simulation Notes, no. 345

[14] O.V. Mikheev, S.A. Podosenov, K. Yu. Sakharov, A.A. Sokolov, Ya.G. Svekis and V.A. Turkin, "New method for calculating pulse radiation from an antenna with a reflector", submitted to IEEE Transactions on Electromagnetic Compatibility

1. The "Sensor and Simulation Notes" can be obtained from the Phillips Laboratory, Kirtland $A F B, N M$ 87117. USA
Dit artikel werd eerder gepubliceerd in:

Second International Conference on the detection of abandoned land mines.

Conference Publication No.458.

IEE, 12-14 October 1998, Edinburgh. 